10

16

\title{
The Landscape of Mutations in Human Fumarate Hydratase
}

\section{David Shorthouse ${ }^{1}$, Michael W J Hall, ${ }^{1,2}$ Benjamin A Hall ${ }^{1 *}$}

${ }^{*}$ Correspondence to be addressed to B.A.H (bh418@mrc-cu.ac.uk)

${ }^{1}$ MRC Cancer Unit, University of Cambridge, Cambridge, CB2 0XZ, UK

${ }^{2}$ Wellcome Trust Sanger Institute, Hinxton, CB10 1SA, UK

6

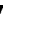

8

9

1

2

3

4

5




\section{ABSTRACT}

27 Fumarate Hydratase (FH) is an enzyme of the citric acid (TCA) cycle that is

28 responsible for reversibly catalysing the conversion between fumarate and malate.

$29 \mathrm{FH}$ loss and subsequent buildup of the oncometabolite fumarate causes hereditary

30 leiomyomatosis and renal cell carcinoma. We explore the mutational landscape of

$31 \mathrm{FH}$ in silico, predict the functional effects of many already detected mutations, and

32 categorise detected but un-characterised mutations in human populations. Using

33 mutational energy predicting tools such as Rosetta and FoldX we accurately predict

34 mutations and mutational hotspots with high disruptive capability. Furthermore,

35 through performing molecular dynamics simulations we show that hinge regions of

36 the protein can be stabilized or destabilized by mutations, with new mechanistic

37 implications of the consequences on the binding affinity of the enzyme for its

38 substrates. Finally, we categorise all potential mutations in $\mathrm{FH}$ into functional groups,

39 and predict which known mutations in the human population are loss-of-function, and

40 therefore predispose patients to papillary renal carcinoma - we validate our findings

41 through analysis of metabolomics data of characterized cell lines.

42 (169 words)

47 Keywords: Mutation, Mutational Screen, Fumarate Hydratase, Molecular Dynamics, 48 Metabolomics 


\section{INTRODUCTION}

55 Fumarate hydratase $(\mathrm{FH})$ is a member of the tricarboxylic acid (TCA) cycle occurring

56 in the mitochondria, and enzymatically metabolises fumarate within the cytosol. $\mathrm{FH}$

57 activity in the cell is responsible for the reversible conversion of the metabolite

58 fumarate into malate, and the knockout or mutational inactivation of $\mathrm{FH}$ in kidneys is

59 linked to an oncogenically-associated buildup of fumarate(1,2). As a result the

60 enzyme $\mathrm{FH}$ has been described as a tumor suppressor, and fumarate as part of a

61 novel classification of molecules named "oncometabolites". Precisely how the

62 buildup of fumarate can be oncogenic is unknown, but recent work points towards suppression of DNA repair responses, EMT, and promotion of mitotic entry upon

64 fumarate buildup(3-5).

Understanding the effects of mutations on the activity and assembly of $\mathrm{FH}$ is of importance for the understanding and stratification of germline mutations in $\mathrm{FH}$, which can predispose patients with a single mutated or deleted allele to hereditary leiomyomatosis and renal cell cancer (HLRCC) upon mutational inactivation of their remaining wild-type copy $(6,7)$. Previous work has identified mutants linked with inherited and de-novo FH-related conditions, including cancer(8) - most notably, the $\mathrm{FH}$ mutation database represents a comprehensive list of mutations and their effects, if known, on FH activity(9).

In recent years numerous methods have been developed for estimating the effects of single point mutations (SNPs) on the stability of a protein structure in silico. Notable methods include FoldX $(10,11)$, which uses an empirical force field to predict the alterations in a protein induced by mutation, and methods included as part of the Rosetta suite $(12,13)$, which uses Monte-Carlo based dynamics to predict energetic effects of mutations. Additionally, molecular dynamics can be used to more comprehensively investigate mutant protein structure, though at significantly higher computational cost. With the advent of high-throughput methods such as CRISPR screening, and larger projects being undertaken to screen populations for mutations and disease, coupled with large-scale disease-focussed data generating projects such as The Cancer Genome Atlas (TCGA)(14) and the International Cancer 
86 functionally relevant mutations from synonymous to detect highly mutated genes

87 exist in the form of statistical tests such as DN/DS(16), mutsig(17), and

88 oncodrive(18), including some methods that take into account structure of the protein

89 such as Rhapsody(19), there is scope for detailed, structure-informed, chemically

90 aware methods to classify mutations, including those not yet observed, into Loss-of-

91 Function (LOF) and benign categories.

92 Here we computationally screen and classify every potential mutation in the available

93 fumarate hydratase structure to study the landscape of potential mutations. We

94 consider the structural and biological implications of each mutation, and thus can

95 predict mechanistic details of every potential mutant. We confirm that our method

96 predicts known functionally relevant mutations, and predict from existing databases

97 of mutations which have an unknown effect, which of them will be damaging to the activity of $\mathrm{FH}$. Overall we predict that $66 \%$ of all mutations to $\mathrm{FH}$ influence activity or assembly. We further validate our predictions through studying the Cancer Cell Line Encyclopaedia (CCLE) $(20,21)$ and show that previously unstudied mutations that we predict to be damaging to the function of $\mathrm{FH}$ result in altered metabolite levels expected from disruption to the activity of $\mathrm{FH}$. 


\section{RESULTS}

\section{Evidence of mutational clustering in FH}

117 Human $\mathrm{FH}$ is formed as a homotetramer of subunits generated from the $\mathrm{FH}$ gene.

118 Each subunit contains 3 domains, Domain 1, Domain 2, and Domain 3 (D1, D2, and

119 D3 respectively) (Fig 1 A). D1 is formed from residues in the range 49-188, D2 is

120 formed from residues in the range 189-439, and D3 from residues in the range 440-

121 510. The full functional protein is an assembly of 4 subunits and contains 4 identical

122 binding pockets made of interactions between 3 subunits (Fig 1 B). There are two

123 proposed regions of importance for catalysis of the fumarate/malate conversion; Site

$124 \mathrm{~A}$, the known active site (hereafter referred to as the binding site), and Site $B$, a

125 region of proposed but unknown functional importance $(22,23)$. For this study we

126 chose to only include the known catalytic site, Site A, defined as residues HIS176,

127 ASN182, SER186, SER187, ASN188, THR234, HIS235, LYS371, VAL372, ASN373,

128 and GLU378 (Fig 1 C). We do not consider Site B due to the unknown and

129 conflicting evidence surrounding its importance. For this study we chose to focus on

130 the crystal structure $5 \mathrm{upp}(24)$, which covers residues $49-510$ of the 510 residue

131 protein assembled into a homotetramer.

132 To study mutations known or suspected to have roles in human disease, we

133 investigated the Fumarate Hydratase Mutation Database(9), which contains 378

134 mutations, including 113 that are distinct missense, at the time of this study. The

135 Fumarate Hydratase Mutation Database attempts to pool all observed mutations in

$136 \mathrm{FH}$, including those that are benign, and a large number of mutations have no clinical

137 or functional annotation. Mutations that are not known to be benign (i.e those either

138 labelled as loss-of-function, or those which are uncharacterised) are shown in Fig 1

139 D.

140 We applied the NMC clustering method to look for clustering of mutations across the

141 1D sequence of the protein(25). We chose to include the top 5 predicted clusters,

142 ranked by significance, and with a size less than 50 residues long. We find the most

143 significant clusters are all within the region of the more prevalent mutations in

144 residues 230 and 233 , indicating that this region is statistically highly over mutated,

145 and potentially a mutationally vulnerable site. 
bioRxiv preprint doi: httos://doi org/101101/852392: this version posted Januarv 312020 . The copvriaht holder for this preprint (which was not certified by peer review) is the author/funder, who has granted bioRxiv a license to display the preprint in perpetuity. It is made available under aCC-BY 4.0 International license.

146

147

148

149

150

151

152

153

154 

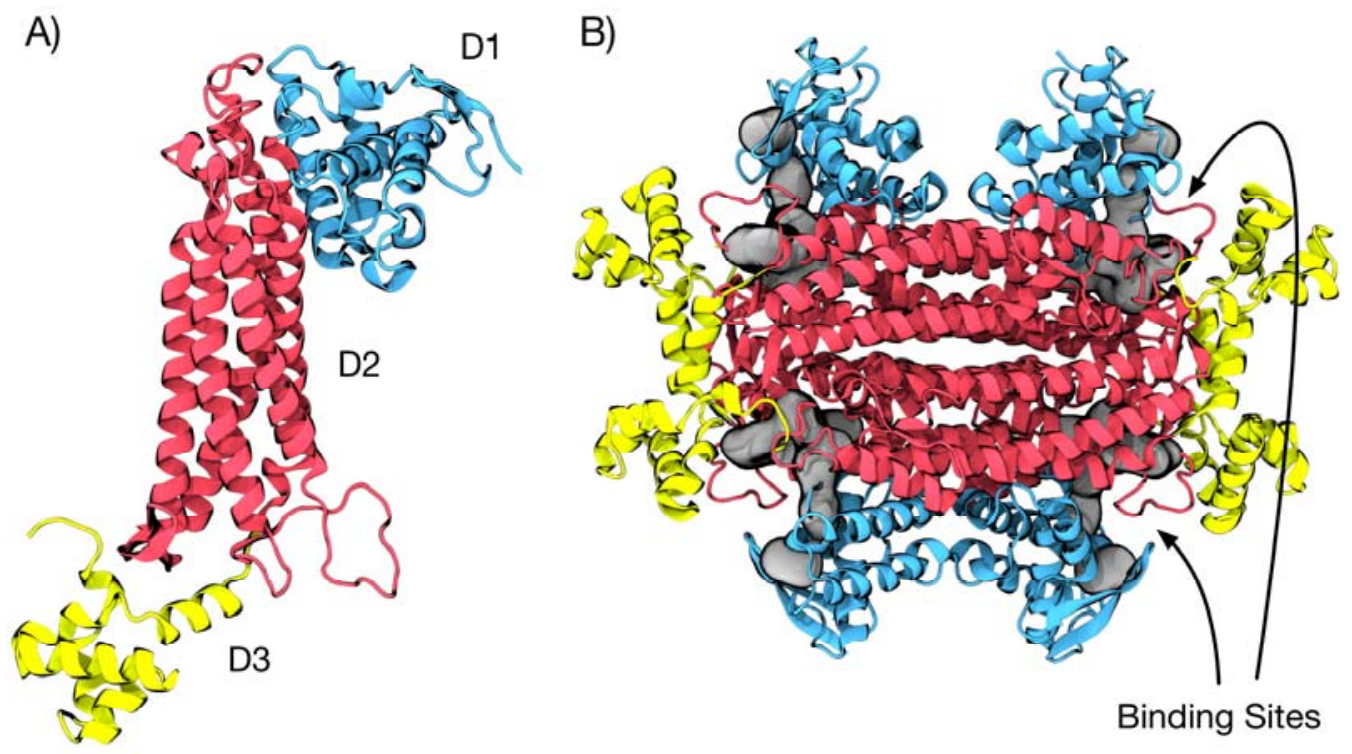

C)

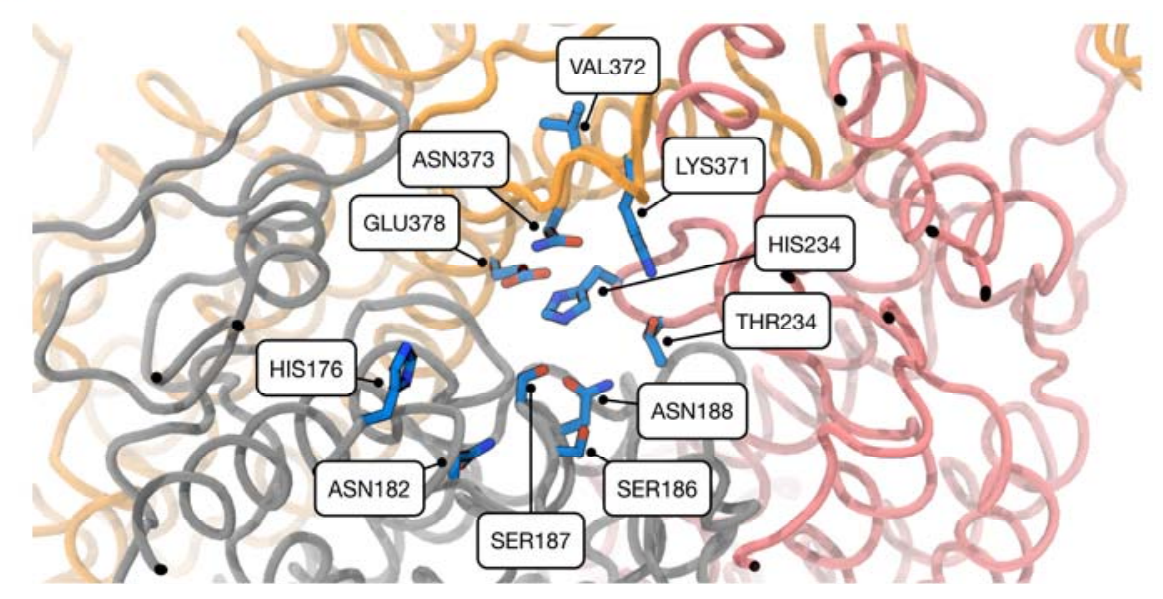

D)

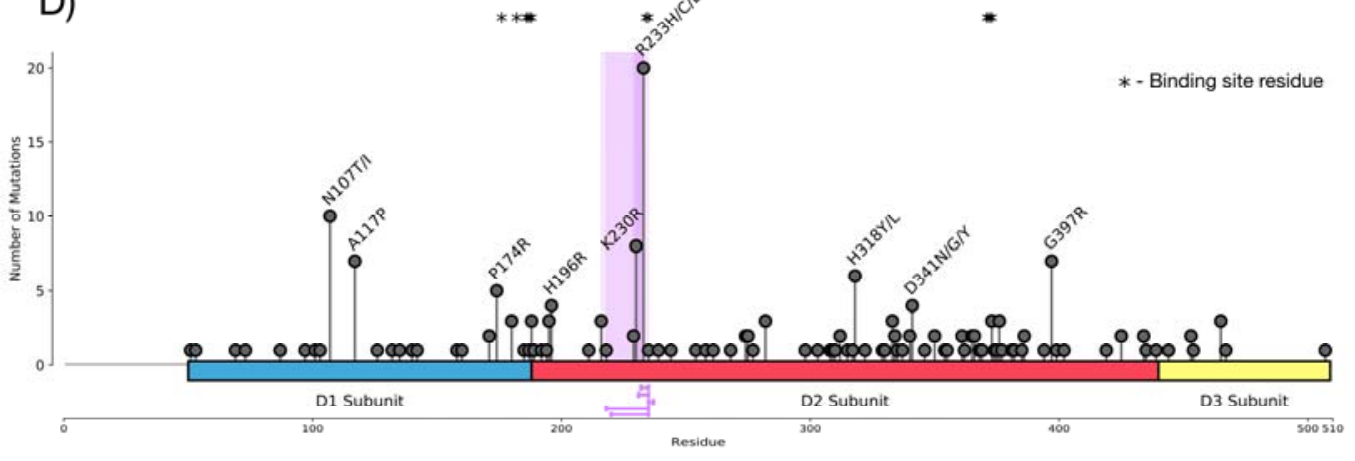

Figure 1: Structure and current mutations in Fumarate Hydratase. A) Structure of a single subunit of $\mathrm{FH}$ showing the D1, D2, and D3 regions. B) Structure of an assembled homotetramer of $\mathrm{FH}$. Binding sites are highlighted and made up on an interface between 3 subunits. C) Close up of the binding site of $\mathrm{FH}$ showing the residues involved in catalytic activity. D) Mutational spectrum of non-benign SNPs in FH. D1, D2, and D3 regions are highlighted in blue, red, and yellow respectively. 
162 Stars indicate residues involved in catalytic activity that make up the binding site of

$163 \mathrm{FH}$. Purple highlight and lines represent the top 5 mutational clusters as calculated by the NMC algorithm.

\section{Classification of mutations by proximity to the binding site and protein hinges}

Residues of the catalytic site in FH have been previously identified as essential for the conversion of fumarate to malate. We define binding site-associated residues as those with alpha-carbons (CA) within $6 \AA$ of the $C A$ of any binding site residue. Mutations this close to the binding site are likely to disrupt the assembly of the binding site, and we classify them as potentially disruptive. A significant number of known mutations are binding-site adjacent (Fig 2 A) and significantly mutated.

We surmised that regions involved in the "hinging" of $\mathrm{FH}$ domains may influence the binding site assembly due to the proximity and reliance of the quaternary structure of multiple domains to make up the binding pocket. We used Gaussian Network Modelling (GNM) within prody $(26,27)$ to predict hinge residues between the subdomains of the protein (Fig 2 B). We used the second normal mode for calculation of hinge residues, as the first normal mode reflects an unphysiological bending of the centre of the protein. Calculating the hinge residues results in residues 196, 198, 232, 242, 270, 317, 401, 411, and 448 being the most likely "hinge points" in the structure, these residues are shown on a single subunit of $\mathrm{FH}$, coloured by eigenvector direction in Fig 2 C. To assess how mutations to this region disrupt the quaternary structure of $\mathrm{FH}$, we chose the simulate the known $\mathrm{R} 233 \mathrm{H}$ mutant, and the wild type (WT) tetrameric assemblies for 200ns each using molecular dynamics simulations. Measuring the angles between CA atoms of two residues in the centre of the D2 and D3 regions with respect to the hinge reveals that the $\mathrm{R} 233 \mathrm{H}$ mutant reduces the angle of the domains by an average of 8 degrees, and so leads to a partial occlusion of the catalytic site of FH (Fig 2 D). From this evidence we conclude that disruption of these hinges are likely to alter the binding site and assembly of $\mathrm{FH}$ - and are likely pathogenic. We chose to treat all mutations with CA atoms within $6 \AA$ of any hinge residue as potentially LOF through disruption of the protein quaternary structure.

192 Overall, we infer that mutations near to either the binding site, or a hinge region of 193 the protein are likely to disrupt or alter the protein function. We find that, from the $\mathrm{FH}$ 
194 mutation database, a significant proportion of mutations can be classed as either

195 binding site-associated, or hinge-associated, including a number of known loss-of-

196 function (LOF) variants. Whilst 42 residues in the 461 amino acid protein structure

$197(9 \%)$ are classified as being "binding site-associated", we find that 11 of the 30 (36\%)

198 known LOF mutations are within these residues, showing a clear bias towards

199 binding site-associated mutations. Similarly, 55 of the $461(12 \%)$ amino acids in the

200 protein structure are classified as "hinge-associated", and we find 7 of the 30 (23\%)

201 within the FH mutation database fulfil this classification, showing a lesser, but still

202 large occurrence bias. Distance calculations for all potential mutations are included

203 in Table S1. 


\section{A)}

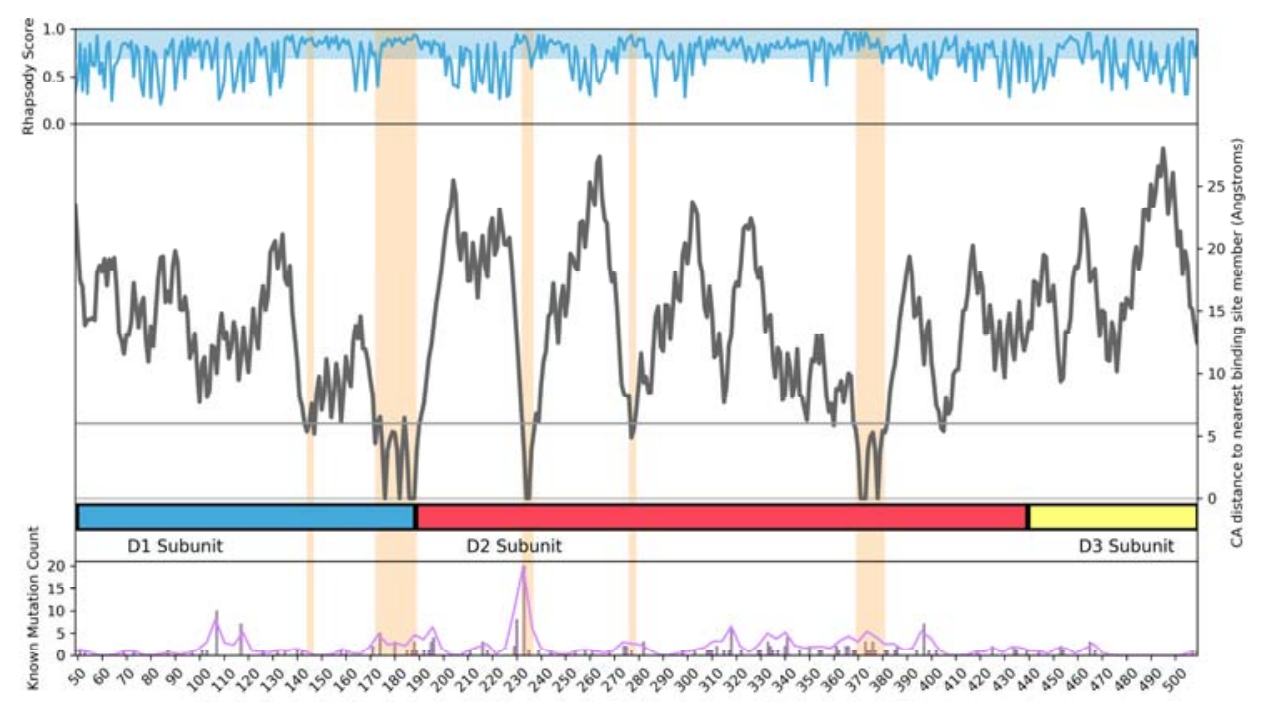

B)

C)
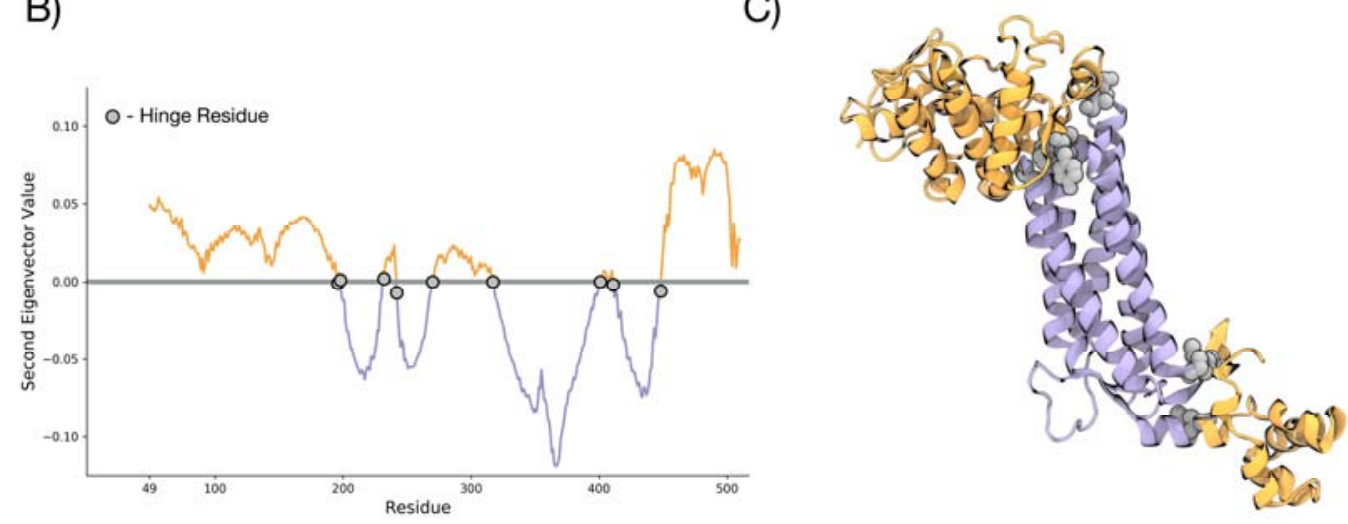

D)

Figure 2: Mutations can be categorised on proximity to functional regions of $\mathrm{FH}$. A)

Alpha carbon (CA) distance from a binding site residue. Shown is: Top: average rhapsody score for each residue, Middle: distance of each residue from a binding site residue by CA distance, Bottom: mutational frequency for each residue. Orange highlights show some regions have high rhapsody scores, low binding site distance, and high mutational frequency. B) Second normal mode eigenvectors per residue for 
211 a single subunit of $\mathrm{FH}$. Residues with an eigenvector above the line are moving

212 generally opposed to those with an eigenvector below the line. Predicted hinge

213 residues are shown in grey. C) Single subunit of $\mathrm{FH}$ coloured according to

214 eigenvector direction (positive as orange and negative as purple). Hinge residues

215 are highlighted as grey. D) Molecular Dynamics simulations of hinge mutations

216 shows altered hinge flexibility. Left: Schematic of the angle measured in each

217 simulation, Right: Angle of WT (red), and R233H mutant FH (blue) over a 200 ns

218 equilibrium molecular dynamics simulation.

\section{High-Throughput mutational stability screen of FH in silico}

220 To study how mutations that are not near the binding site or hinge regions may have

221 effects on the structure of the protein, we sought to generate predicted mutational

222 energy changes $(\Delta \Delta \mathrm{G})$ for every potential amino acid substitution in the $\mathrm{FH}$

223 structure. We chose to use two conceptually different methods and use an average

224 between the two methods to study each potential mutant. We used the FoldX

225 method(10, 11), and the Rosetta cartesian_ddg method(12, 13), (hereafter described

226 as the Rosetta method) to perform mutational energy calculations.

227 To perform mutant calculations, the pdb structure 5UPP was first relaxed using the

228 FoldX RelaxPDB method, before each mutation and its resultant $\Delta \Delta \mathrm{G}$ was

229 calculated. We additionally calculate the Relative Solvent Accessible Surface Area

230 (RSA) for each wild-type (WT) residue. Mutations on the surface of the protein are

231 unlikely to dramatically alter the folding of the protein, so we chose to only consider a

232 mutation potentially destabilizing if it is buried, defined as having an RSA $<=0.2$.

234 We find a good agreement between the FoldX and Rosetta methods, with an $r$ of

$2350.67(p<0.0001)$ for all mutational energies (Fig 3 A). This correlation is good given

236 previous reports showing an overlap between Foldx and Rosetta-ddg of only 12\%-

$23725 \%$ when considering stabilizing mutations (28). Notably however, both methods

238 appear to agree on predictions of mutations with extremely high energy, but there is

239 a significant portion of the distribution that shows a reasonably poor correlation,

240 particularly mutations that have a predicted $\Delta \Delta \mathrm{G}$ between 1 and $-1 \mathrm{kcal} / \mathrm{mol}$. We

241 additionally chose a cutoff of $2.5 \mathrm{kcal} / \mathrm{mol}$ to classify mutations as destabilizing, as 
242 this provides a good separation between known loss of function and known benign

243 mutations that are not binding site adjacent (Fig 3 B). Across all potential mutations

244 we find that $\sim 45 \%$ (3968 out of 8778 ) meet this criterion (Fig $3 \mathbf{C}$ ). This fits roughly

245 with historical data of mutational stability in T4 lysozyme, which found that $45 \%$ of

246 mutational sites lead to structural inactivation of enzymatic function (29).

247 Plotting mutational frequency for both methods, and their average for each residue

248 (Fig 4 A) reveals that the most destabilizing mutations predicted by either method

249 are in regions with a large number of buried amino acids, as expected. When plotting

250 these mutations on the structure of the protein (Fig 4 B), we find the most

251 significantly destabilizing mutations are those packed within the centre of D1, and on

252 the interface between D1 and D2. This location suggests mutational disruption will

253 alter the position of the D1/D2 interface, and thus will affect the binding site

254 conformation, whereas mutations within the core D2 region are likely to influence the 255 stability of the fully assembled tetramer.

A)

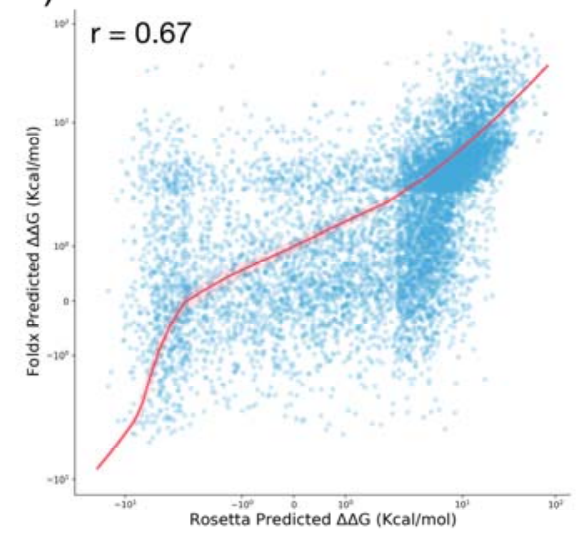

C)

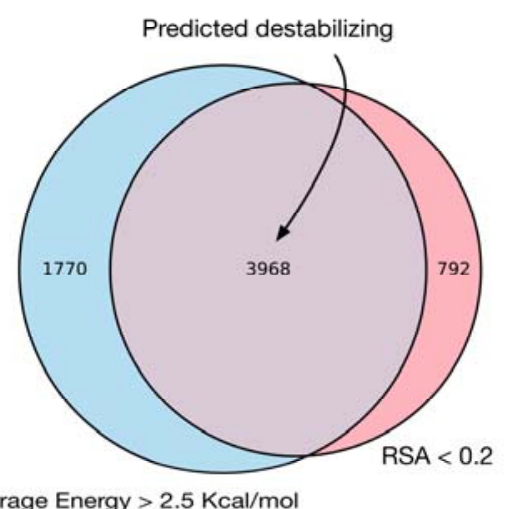

B)

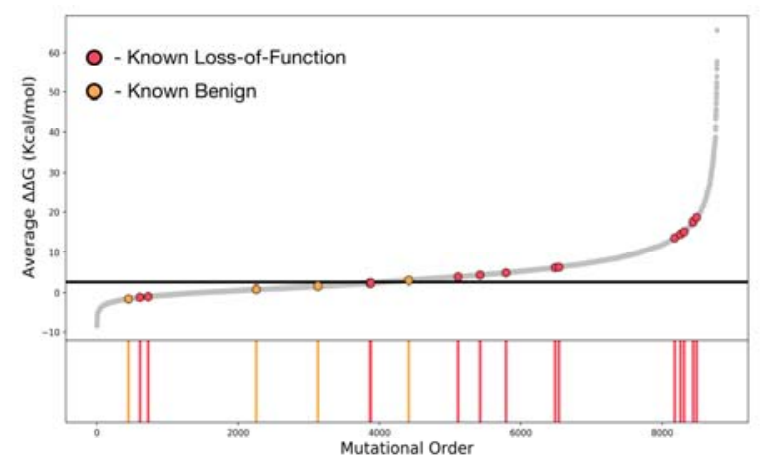


257 Figure 3: Prediction of Destabilizing Mutations. A) Comparison of $\Delta \Delta G$ calculations

258 from FoldX and Rosetta. Correlation $r$ is spearmans rank. B) Position of known loss-

259 of-function (red) and benign mutations (orange) on the $\Delta \Delta \mathrm{G}$ spectrum. Mutations are

260 ordered in acsending mutational $\Delta \Delta \mathrm{G}$. Black line represents $2.5 \mathrm{Kcal} / \mathrm{mol}$ cutoff. C)

261 Overlap between residues with a high predicted mutational energy (Defined as those

262 with average $\Delta \Delta \mathrm{G}>2.5 \mathrm{Kcal} / \mathrm{mol}$ ) and buried residues ( $\mathrm{RSA}<0.2)$. In total 3968

263 mutations are classified as destabilizing by taking the overlap between these two

264 criterion.

A)
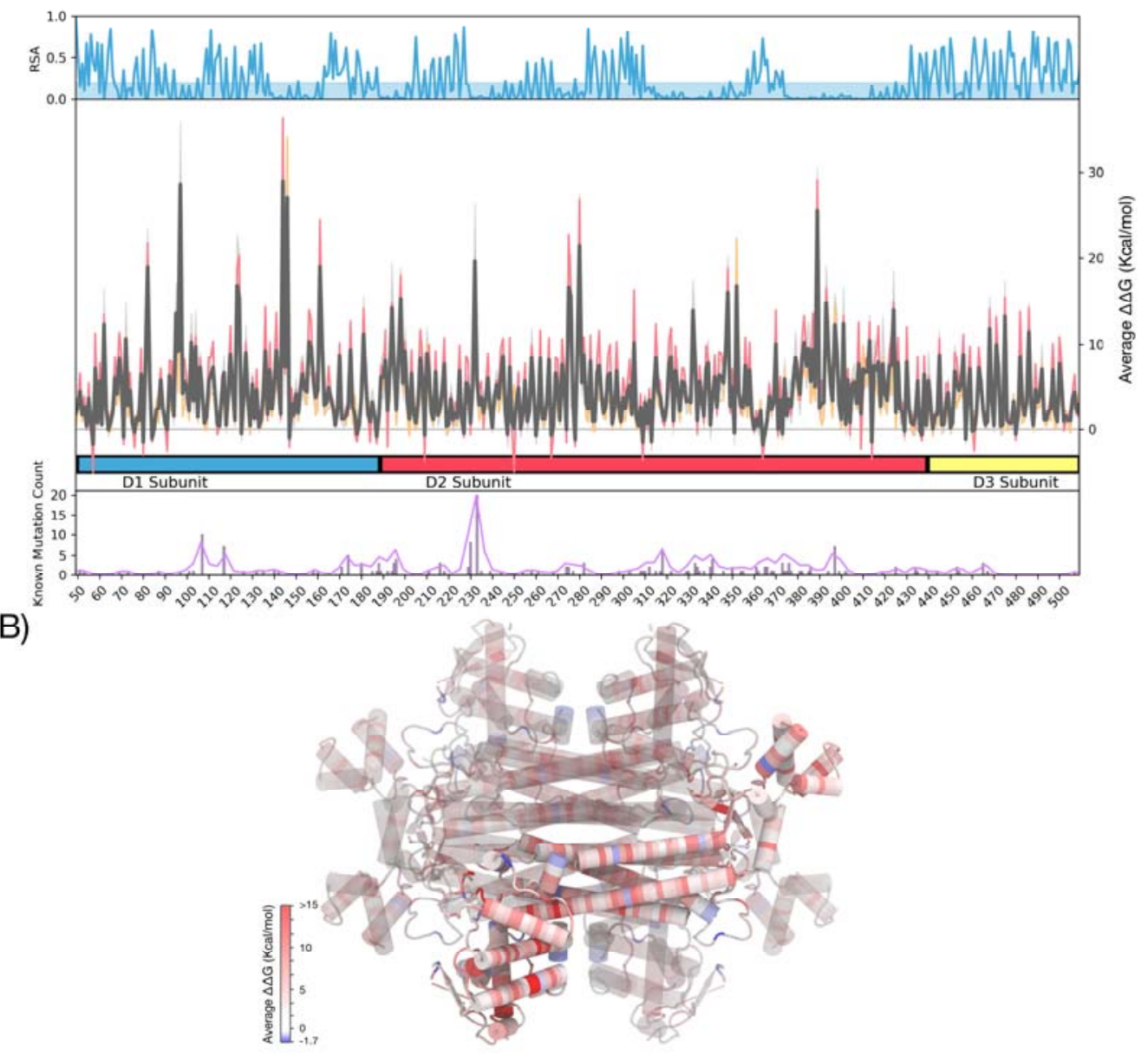

267 Figure 4: Predicted $\Delta \Delta \mathrm{G}$ for every mutation in Fumarate Hydratase. A) Average 268 mutational energy per residue in the FH structure. Top: Relative solvent accesible 
surface area (RSA) for every residue. Blue highlight indicates RSA $<0.2$, classified as buried. Middle: average $\Delta \Delta G$ for each residue (Grey). Red and Orange lines represent average Rosetta and FoldX calculations respectively. Bottom: Mutational frequency from the $\mathrm{FH}$ mutation database for each residue in $\mathrm{FH}$. B) Mutational $\Delta \Delta \mathrm{G}$ applied to the structure 5upp of $\mathrm{FH}$. Red indicates high average $\Delta \Delta \mathrm{G}$, and so represents areas where mutations are likely to disrupt the structure. Blue represents regions of generally stabilizing mutations.

\section{Existing mutations are accurately categorised based on known phenotypic effects}

Overall, we define a scheme for classifying mutations into different categories of potentially disrupting, predicted LOF substitutions (Fig 5 A). The initial structure is relaxed using FoldX, before the binding site and hinge regions are calculated and classified, additionally mutations that are potentially destabilizing are defined based on average energy from the Rosetta and FoldX mutation methods, plus screened for buried mutations through calculating the RSA. This results in a categorisation for every mutation, where is it classified as predicted silent, binding site, hinge site, or destabilizing (including combinations of disruptive mutation types) (Table S1). Overall we classify 5811 out of $8778(66 \%)$ mutations as potentially functionally disruptive, similar to a study of mutational effects on TP53, which found that roughly $50-60 \%$ of all possible mutations were functionally disruptive(30).

We compared our predictions with known mutations in the $\mathrm{FH}$ mutations database.

We predicted a classification for all mutations within the database and compared this to a classification for each categorised as loss of function (LOF), benign, or of unknown functional effect. In total 34 mutations had a known (or implied) functional effect, whilst 73 were classified as unknown (Table S2). We find that 24 out of 30 (80\%) mutations are correctly classified as LOF using our classification scheme, and 3 out of $4(75 \%)$ are correctly classed as benign (Fig 5 B). Of the mutations incorrectly classified as benign when they are known to be LOF, two mutations involve cysteine (C434Y, Y465C), which is known to be modelled poorly by Rosetta cartesian_ddg, The mutation incorrectly classified as deleterious when it is listed as 
300 benign within the $\mathrm{FH}$ mutation database is R268G. We predict the R268G mutation

301 to be both destabilizing $(\Delta \Delta \mathrm{G}>2.5 \mathrm{kcal} / \mathrm{mol}, \mathrm{RSA}<0.2)$ and hinge-associated.

302 Whilst the mutation is listed as benign, no experimental information is cited, and

303 PolyPhen-2(31), and Rhapsody also classifiy this particular mutation as damaging,

304 indicating that the benign classification for this mutation may be questionable. We

305 ran a molecular dynamics simulation of the R268G mutant. Simulations predict that

306 mutant R268G reduces the hinge angle of the D1/D2 domains by $\sim 5$ degrees (Fig

307 S1), and supports previous evidence from the R233H mutant, that hinges within he 308 protein can effect binding site assembly. Of the 73 unknown mutations, we predict

309 that 28 are functionally benign, and 45 are potential LOF mutations.

A)

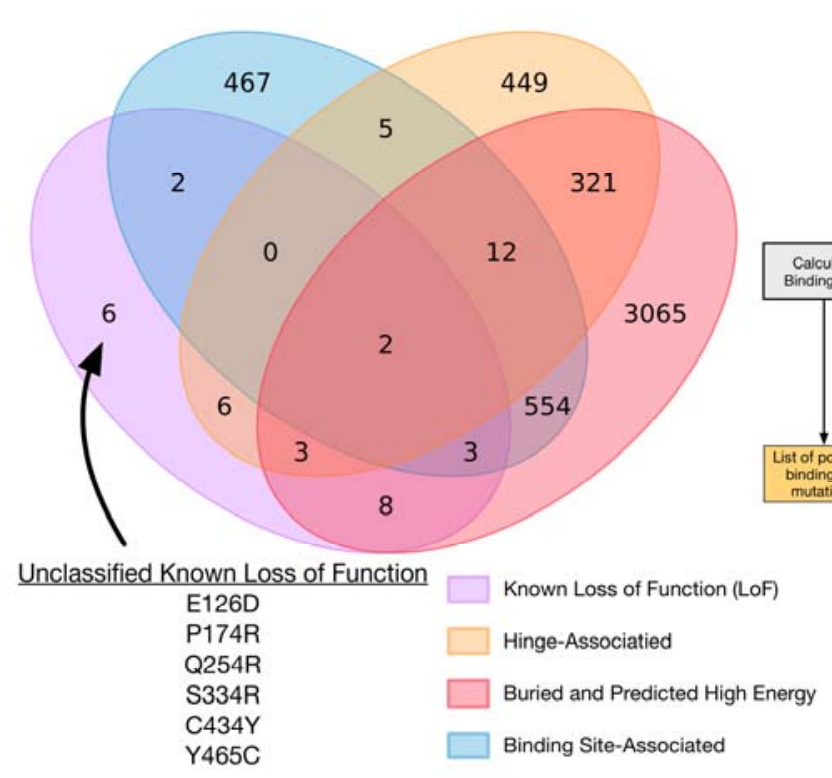

B)

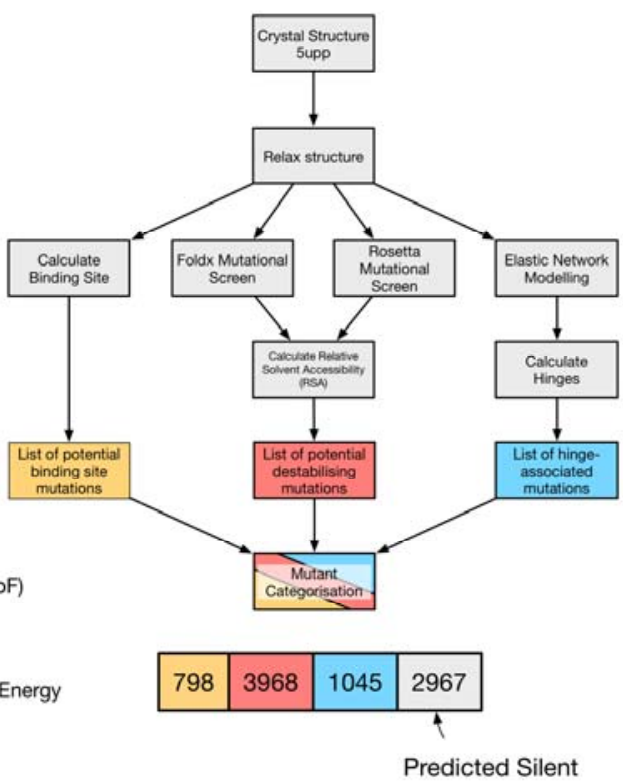

311 Figure 5: Prediction of known Loss-of-Function (LOF) mutations. A) Venn diagram

312 showing overlap between Hinge-associated (orange), destabilizing (red) and Binding 313 site-associated (blue) mutations. 30 known LOF mutations are included (purple) 24 314 mutations are correctly categorized as LOF, whilst 6 are incorrectly categorized as 315 benign mutations. B) Schema for categorization of mutations in $\mathrm{FH}$. The structure is 316 initially relaxed using FoldX RelaxPDB, residues within $6 \AA$ of the binding site are 317 calculated resulting in a list of 798 binding site associated mutations (orange). FoldX 318 and Rosetta are used to calculate the $\Delta \Delta \mathrm{G}$ for every mutation and this is subset by 319 the relative solvent acessible surface area resulting in 3968 potentially destabilizing 
320 mutations (red). Elastic network modelling is performed to generate hinge regions of

321 the protein, and residues within $6 \AA$ of hinges are calculated, resulting in 1045 hinge

322 associated mutations (blue). 2967 mutations are predicted to be silent.

\section{Mutations with unknown properties can be accurately predicted to be}

\section{4 functional or neutral}

325 To visualise all potential mutations in $\mathrm{FH}$ we chose to plot all mutations using 326 umap(32). Umap attempts to cluster items by similarity, in a manner similar to 327 prinical component analysis, or TSNE (33). We ran umap on all mutations using the 4 major axes involved in the classification - Minimum distance to a binding site residue, minimum distance to a hinge residue, average $\Delta \Delta \mathrm{G}$ of mutation, and RSA for each residue (Fig 6 A). We find that distinct regions of the plot cluster into functionally different mutations when coloured by classification. There is a region specifically for hinge-associated mutations, binding site-associated, and unknown (not predicted damaging) mutations. In particular, the region of "unknown" (not classified as damaging) mutations overlaps significantly with a number of predicted destabilizing mutations, indicating that discrimination between these mutations is difficult, and perhaps not accurate with currently available data. We find that most of the benign mutations, aside from R268G are found clearly within the regions of predicted benign mutations. R268G clusters with the hinge mutation region as expected from our previous classification. For the known LOF mutations, we find they mostly cluster within the well defined regions for binding site, hinge, and destabilizing mutations. There are some mutations, particularly those which were misclassified, that fall within ambiguous regions of state space in the mutational landscape, and so are hard to classify using our defined criterion.

To test the predictive power of our classification scheme we used the Cancer Cell Line Encyclopedia to look for changes in metabolite levels associated with mutations in $\mathrm{FH}(21,34)$. We find 42 mutations (35 unique) in $\mathrm{FH}$ within 34 individual cell lines (Table S3). Selecting only for missense mutations yielded 25 mutations (20 unique) within 23 unique cell lines. We classified the mutations according to our criterion as 350 either predicted LOF, or predicted benign. We find that by analysis of metabolomics 351 data included in the CCLE database, mutations that we predict to be LOF have a 
bioRxiv preprint doi: https://doi.org/10.1101/852392; this version posted January 31, 2020. The copyright holder for this preprint (which was not certified by peer review) is the author/funder, who has granted bioRxiv a license to display the preprint in perpetuity. It is made available under aCC-BY 4.0 International license.

352 higher average level of fumarate/mateate/alpha-ketoisovalerate detected in media

353 than cells with predicted benign mutations $(p=0.035)$ - indicating that these cell

354 lines may have an accumulation of fumarate as a result of inactive levels of $\mathrm{FH}$ (Fig 3556 B,C).

A) Predictions - Unknown Mutations

Hinge-Associated
Destabilizing
Binding Site-Associated
Silent

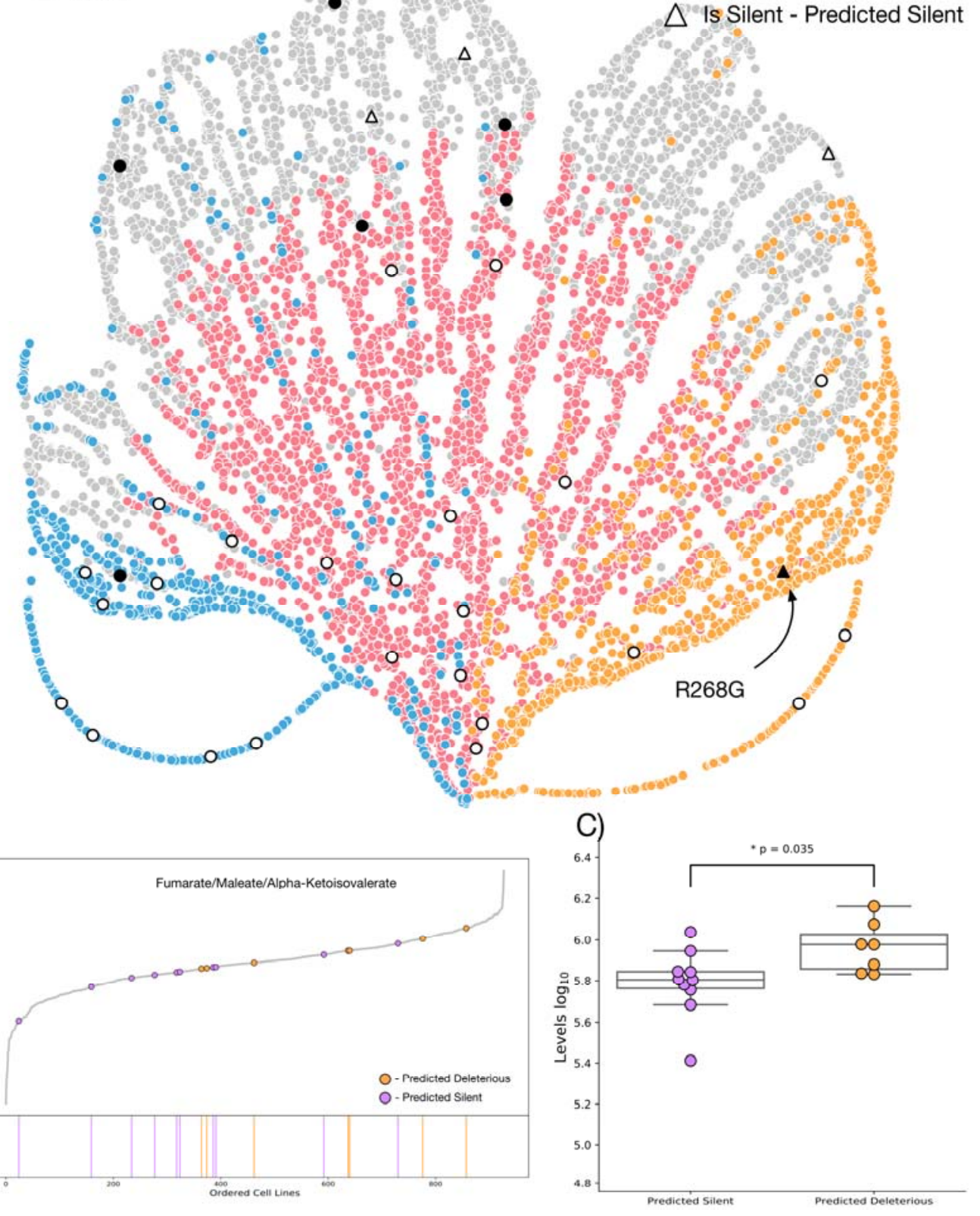

B)

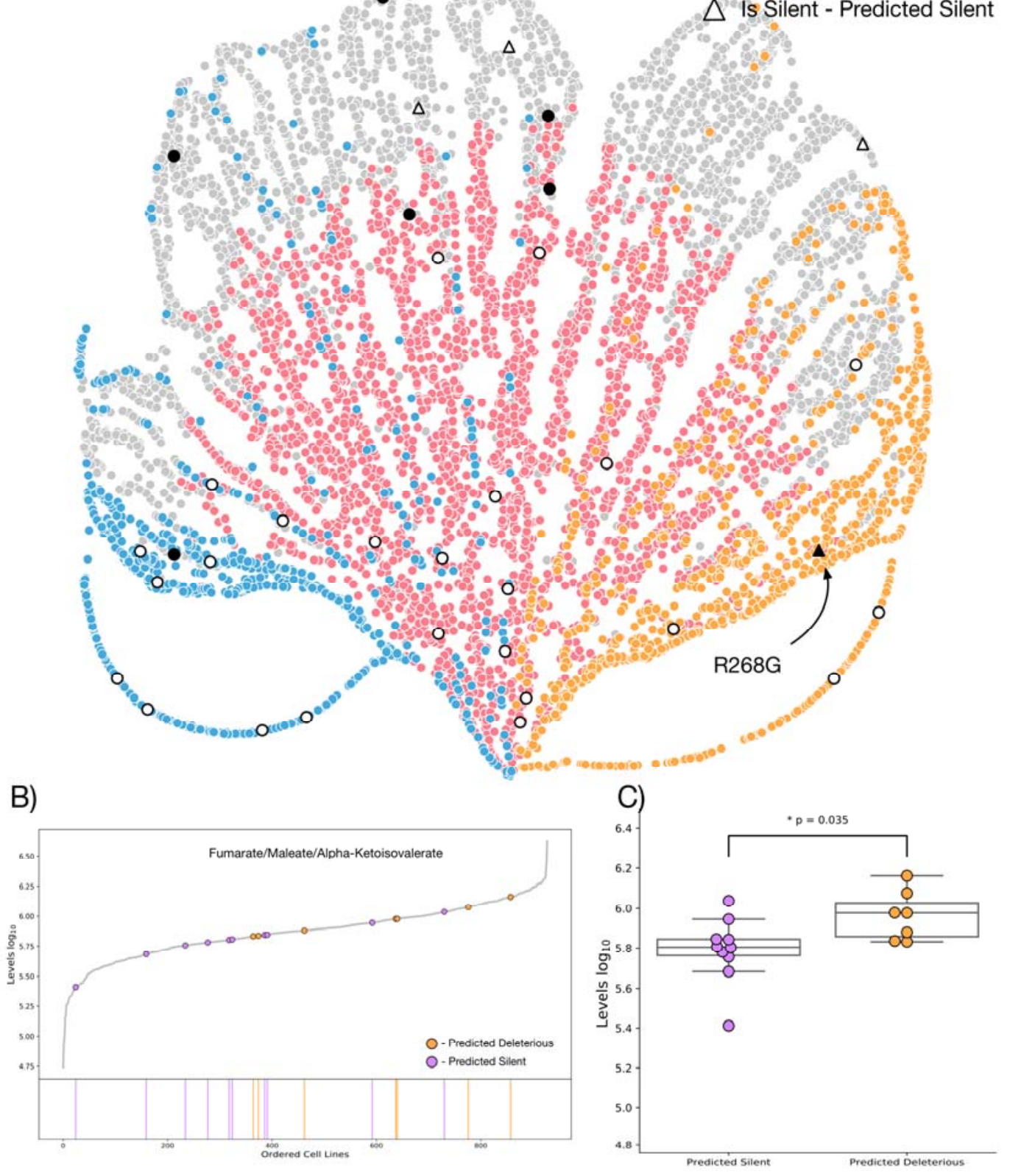

Predictions - Known Mutations

Is LoF - Predicted LoF

- Is LoF - Predicted Silent

$\Delta$ Is Silent - Predicted LoF

$\triangle$ is Silent - Predicted Silent

356 
357 Figure 6: Mutational Landscape of Fumarate Hydratase. A) Umap for all mutations

358 in $\mathrm{FH}$. Mutations are coloured by classification. Hinge-associated (orange),

359 Destabilizing (red), and Binding site-associated (blue) are shown clustered into

360 groups. Predicted silent mutations (grey) are also shown. Overlayed are our

361 predictions for characterized mutations in the $\mathrm{FH}$ mutation database. Mutations that

362 are known Loss-of-Function (LOF) are circular and coloured according to whether

363 we predict them to be LOF (black) or silent (white). Known benign mutations are in

364 triangles, and also coloured according to whether we predict them to be LOF (black)

365 or silent (white). The questionable known benign mutation R268G is labelled B)

366 Mutations in the Cancer Cell Line Encyclopedia (CCLE) metabolomics data. All cell

367 lines are ranked according to their detected levels of Fumarate/Maleate/Alpha-

368 Ketoisovalerate. Coloured are cell lines with mutations in $\mathrm{FH}$ that we predict to bo

369 LOF (orange), or silent (purple). C) Swarm plot for levels of

370 Fumarate/Maleate/Alpha-Ketoisovalerate in mutant FH cell lines. Mutations predicted

371 to be silent are significantly lower than mutations predicted to be LOF ( $p$ value

372 represents independent T test). 


\section{DISCUSSION}

In conclusion, we have shown, using a comprehensive combination of techniques, that we can categorise accurately the functional effects of any potential missense mutation in $\mathrm{FH}$. Beyond $\mathrm{FH}$, we present an integrated series of methods that can be adapted for mutationally screening any protein for functionally relevant mutations in a reasonably small amount of computational time. Our workflow predicts the functional effects of all mutations that can be compared to existing methods based on machinelearning principles such as rhapsody and polyphen, at significantly lower time and effort expenditure than experimental characterization. Whilst some other methods incorporate some manner of structural analysis in their predictions, ours demonstrates a new perspective, as it explicitly models every potential mutation in a structure, allowing it to interface directly with other computational techniques in the field such as molecular dynamics simulations to further study mutations of interest.

Biologically we propose three ways in which mutations can potentially disrupt the catalytic activity of $\mathrm{FH}$, in particular we find that addition of hinge altering mutations are necessary for classification of many known LoF mutations, indicating that there is a biological relevance, and hinting at a mechanism for, mutations that change the flexibility and stiffness of protein hinges in this case. Additionally, we chose to exclude site $B$ from our analysis of mutation disruption and find that we are able to classify almost all known mutations without its inclusion. This implies that mutations in site B may not have functional or disease-related relevance, despite some evidence that site B can alter catalytic activity of the enzyme(35). This is reinforced by the fact that 27 of the 461 residues within the protein structure are classified as near site $\mathrm{B}(6 \%)$, and only 3 of 30 residues in the $\mathrm{FH}$ mutation database (10\%) are near to site $B$, showing a poor to negligible enrichment of mutations in site $B$ when compared to similar calculations for site A.

Fumarate hydratase represents a good first-use case for high-throughput mutational screen due to the need to understand mutations in their functional context, but as 
413 mutational detection techniques, and high-throughput mutational studies increase

414 the need to be able to classify mutations confidently as benign and LOF is more

415 important. Here we show that our method accurately classifies known LOF and

416 benign mutations with a high degree of accuracy, and predict which mutations

417 discovered in the human population are likely to have functional relevance, and

418 therefore predispose patients to particular metabolic diseases.

Whilst the accuracy of our method with the current data is high, there are clear regions where the analysis is not able to discriminate between mutations on the borderline between destabilizing and benign, this results from the lack of accuracy in

423 the mutational $\Delta \Delta G$ calculations, despite using the best available methods at time of 424 study(13). As better methods become available it will be of interest to improve upon

425 this work to attempt a more accurate classification.

427 Finally, whilst the work here focusses on a single molecule within the TCA cycle, FH, 428 structural data has existed for a large number of enzymes within the cycle for some 429 time(36-39), and it would be of great interest to look into mutations across entire 430 metabolic pathways. With this study laying the groundwork, it will be of future interest 431 to model all mutations in all enzymes, and attempt to further link these with genomic 432 and metabolomic data that is already available. Whilst the computational intensity of 433 this work is high, it is feasible to screen thousands of mutations in only a few days of 434 computational time. 


\section{METHODS}

442 All data used in this study, including the code used in generating all figures from raw

443 data is available publicly at: https://github.com/shorthouse-mrc/Fumarate_Hydratase

\section{$444 \quad$ FH mutation database}

445 The FH mutation database was downloaded from the Leiden Open Variation

446 Database(9) (https://databases.lovd.nl/shared/variants/FH/unique). Missense

447 mutations were manually curated into categories (Loss of Function, Benign, and

448 Unknown) based on their implied clinical classification, and variant remarks, which

449 contained information regarding $\mathrm{FH}$ enzymatic activity.

\section{$450 \quad$ Mutational Clustering}

451 Mutational clustering was performed with the NMC clustering algorithm, which

452 attempts to discern the likelihood of a mutation spectrum occurring by random

453 chance. NMC returns clusters of mutations that are statistically significant. We chose

454 to run the NMC algorithm using the R library iPAC(25), using an alpha cutoff value of

4550.05 , and the Bonferroni multiple test correction method (see supplementary code).

\section{Gaussian Network Modelling}

457 GNM was implemented using the Prody package in python(40). A Kirchhoff matrix

458 was constructed using the gnm. buildKirchhoff command with the parameters cutoff =

45910.0 and gamma $=1.0$. Normal modes were then calculated using the

460 gnm.calcModes() command. Predicted hinges were assessed using the

461 gnm.getHinges() command. The full protocol, implemented in a jupyter notebook is

462 available at https://github.com/shorthouse-mrc/Fumarate_Hydratase .

\section{Molecular Dynamics Simulations}

464 Molecular dynamics was performed using Gromacs version 2018.1(41). We chose to

465 simulate proteins using the GROMOS 54a7 forcefield(42). 
466 The protein structure was first repaired using FoldX(10) "RepairPDB" with the

467 following command:

468

469

470

\$foldx --command=RepairPDB --pdb=5upp.pdb --ionStrength=0.05 --pH=7 -vdwDesign $=2$

The protein was then placed in a cubic box size $15 \times 15 \times 15 \mathrm{~nm}$ and solvated with roughly $90,000 \mathrm{spc}$ water molecules. Counterions were introduced to a neutral charge, and to a concentration of $0.05 \mathrm{~mol} /$ litre. The system was energy minimized using the steepest descents algorithm until the maximum force, $F_{\max }$, of the system reached below $1000 \mathrm{~kJ} / \mathrm{mol} / \mathrm{nm}$.

Equilibration was performed using the NVT, followed by the NPT ensembles for 100 ps each. We chose to use the verlet cutoff scheme and PME electrostatics, and utilized periodic boundary conditions in the $\mathrm{x}, \mathrm{y}$, and $\mathrm{z}$ planes.

Molecular dynamics was performed for 200 ns retaining velocities from the NPT equilibration. We used the V-rescale temperature coupling scheme, and ParrinelloRahman isotropic pressure coupling.

\section{FoldX $\Delta \Delta G$ Calculations}

FoldX predicted $\Delta \Delta \mathrm{G}$ was calculated using the PositionScan command within FoldX4. Positionscan was run on each residue in the protein structure sequentially using the following command:

\$foldx --command=PositionScan --pdb=5upp.pdb --ionStrength=0.05 --pH=7 -vdwDesign=2 --pdbHydrogens=false - -positions $=49$

For positionscan on the $49^{\text {th }}$ residue (I think residue in the FH structure 5UPP).

\section{$\underline{\text { Rosetta } \Delta \Delta G \text { Calculations }}$}

Rosetta predicted $\Delta \Delta \mathrm{G}$ was calculated using the cartesian_ddg method as described in Kellogg et al: 
\$path/to/source/bin/cartesian_ddg.static.linuxgccrelease -in:file:s 5upp.pdb in::file::fullatom -database /path/to/database/ -ignore_unrecognized_res true ignore_zero_occupancy false -fa_max_dis 9.0 -ddgccartesian -ddg::mut_file mutfile.txt -ddg:::iterations 3 -ddg::dump_pdbs true -ddg::suppress_checkpointing true -ddg::mean true -ddg::min true -ddg:output_silent true -bbnbr 1 beta_nov16_cart > logfile.log

$490 \Delta \Delta \mathrm{G}$ was calculated by averaging the energy of 3 models of each mutation and 491 comparing it to the WT calculation.

\section{Umap}

494 We used Umap(32) based on the github repository at

495 www.github.com/Imcinnes/unmap

\section{Cancer Cell Line Encyclopedia Data}

497 Cancer Cell Line Encylopedia (CCLE) mutation data was downloaded from the

498 Broad Institute at: https://portals.broadinstitute.org/ccle/data. Metabolomics data 499 was obtained from the supplementary data of Li et al(21).

\section{Data Analysis}

501 Both MDanalysis(43) and Biopython(44) were used for analysis of structural data.

502 Data analysis workflows are available in jupyter notebooks available at 503 https://github.com/shorthouse-mrc/Fumarate_Hydratase .

505 AUTHOR CONTRIBUTIONS

506 DS and BAH conceived the study and wrote the manuscript. DS generated all data 507 and performed all analysis. All authors were responsible for editing of the 508 manuscript. 


\section{ACKNOWLEDGEMENTS}

511 We thank the Frezza group, in particular Christian Frezza for support and

512 constructive feedback during the generation of this manuscript. This work was

513 supported by the Medical Research Council (grant no. MR/S000216/1). M.W.J.H.

514 acknowledges support from the Harrison Watson Fund at Clare College, Cambridge.

515 B.A.H. acknowledges support from the Royal Society (grant no. UF130039).

\section{REFERENCES}

517 1. C. Ewbank, J. F. Kerrigan, K. Aleck, Fumarate Hydratase Deficiency

518 (University of Washington, Seattle, 1993) (October 24, 2019).

2. M. Yang, T. Soga, P. J. Pollard, J. Adam, The emerging role of fumarate as an oncometabolite. Front. Oncol. 2, 85 (2012). involved in DNA double-strand break resection through a functional interaction with Sae2. Curr. Genet. 64, 697-712 (2018). Cytosolic/Nuclear Component of the DNA Damage Response. PLoS Biol. 8, e1000328 (2010).

C. Schmidt, M. Sciacovelli, C. Frezza, Fumarate hydratase in cancer: A multifaceted tumour suppressor. Semin. Cell Dev. Biol. (2019) https:/doi.org/10.1016/j.semcdb.2019.05.002 (November 1, 2019).

534 7. N. A. Alam, S. Olpin, I. M. Leigh, Fumarate hydratase mutations and cancer. Br. J. Dermatol. 153, 11-17 (2005). 
537 8. G. R. Clark, et al., Germline FH Mutations Presenting With Pheochromocytoma. J. Clin. Endocrinol. Metab. 99, E2046-E2050 (2014).

9. J.-P. Bayley, V. Launonen, I. P. Tomlinson, The FH mutation database: an online database of fumarate hydratase mutations involved in the MCUL (HLRCC) tumor syndrome and congenital fumarase deficiency. BMC Med. Genet. 9, 20 (2008).

10. J. Delgado, L. G. Radusky, D. Cianferoni, L. Serrano, FoldX 5.0: working with RNA, small molecules and a new graphical interface. Bioinformatics 35, 4168-

11. J. Schymkowitz, et al., The FoldX web server: an online force field. Nucleic 4169 (2019).

12. R. F. Alford, et al., The Rosetta All-Atom Energy Function for Macromolecular Modeling and Design. J. Chem. Theory Comput. 13, 3031-3048 (2017).

13. A. Strokach, C. Corbi-Verge, P. M. Kim, Predicting changes in protein stability caused by mutation using sequence-and structure-based methods in a CAGI5 blind challenge. Hum. Mutat. 40, 1414-1423 (2019).

14. K. Tomczak, P. Czerwińska, M. Wiznerowicz, The Cancer Genome Atlas (TCGA): an immeasurable source of knowledge. Contemp. Oncol. (Poznan, Poland) 19, A68-77 (2015).

16. I. Martincorena, et al., Universal Patterns of Selection in Cancer and Somatic Tissues. Cell 171, 1029-1041.e21 (2017).

17. M. S. Lawrence, et al., Mutational heterogeneity in cancer and the search for new cancer-associated genes. Nature 499, 214-218 (2013).

18. D. Tamborero, N. Lopez-Bigas, A. Gonzalez-Perez, Oncodrive-CIS: A Method Changes on Expression. PLoS One 8, e55489 (2013). 
565 19. L. Ponzoni, Z. N. Oltvai, I. Bahar, Rhapsody: Pathogenicity prediction of human missense variants based on protein sequence, structure and dynamics.

20. M. Ghandi, et al., Next-generation characterization of the Cancer Cell Line Encyclopedia. Nature 569, 503-508 (2019).

21. H. Li, et al., The landscape of cancer cell line metabolism. Nat. Med. 25, 850860 (2019).

22. S. Picaud, et al., Structural basis of fumarate hydratase deficiency. J. Inherit. Metab. Dis. 34, 671-6 (2011).

23. I. A. Rose, T. M. Weaver, The role of the allosteric B site in the fumarase reaction. Proc. Natl. Acad. Sci. U. S. A. 101, 3393 (2004).

24. M. A. Ajalla Aleixo, V. L. Rangel, J. K. Rustiguel, R. A. P. de Pádua, M. C. Nonato, Structural, biochemical and biophysical characterization of recombinant human fumarate hydratase. FEBS J. 286, 1925-1940 (2019).

25. J. Ye, A. Pavlicek, E. A. Lunney, P. A. Rejto, C. H. Teng, Statistical method on nonrandom clustering with application to somatic mutations in cancer. BMC Bioinformatics (2010) https:/doi.org/10.1186/1471-2105-11-11.

26. T. Haliloglu, I. Bahar, B. Erman, Gaussian Dynamics of Folded Proteins. Phys. Rev. Lett. 79, 3090-3093 (1997).

27. I. Bahar, A. R. Atilgan, B. Erman, Direct evaluation of thermal fluctuations in proteins using a single-parameter harmonic potential. Fold. Des. 2, 173-181 (1997).

28. O. Buß, J. Rudat, K. Ochsenreither, FoldX as Protein Engineering Tool: Better Than Random Based Approaches? Comput. Struct. Biotechnol. J. 16, 25-33 (2018).

29. D. Rennell, S. E. Bouvier, L. W. Hardy, A. R. Poteete, Systematic mutation of bacteriophage T4 lysozyme. J. Mol. Biol. 222, 67-88 (1991). 
592 30. E. Kotler, et al., A Systematic p53 Mutation Library Links Differential Functional Impact to Cancer Mutation Pattern and Evolutionary Conservation. Mol. Cell

31. I. A. Adzhubei, et al., A method and server for predicting damaging missense mutations. Nat. Methods 7, 248-249 (2010).

32. L. Mclnnes, J. Healy, J. Melville, UMAP: Uniform Manifold Approximation and Projection for Dimension Reduction (2018) (November 7, 2019).

33. L. van der Maaten, G. Hinton, Visualizing Data using t-SNE. J. Mach. Learn. Res. 9, 2579-2605 (2008).

34. J. Barretina, et al., The Cancer Cell Line Encyclopedia enables predictive

35. N. A. Alam, et al., Missense Mutations in Fumarate Hydratase in Multiple Cutaneous and Uterine Leiomyomatosis and Renal Cell Cancer. J. Mol. Diagnostics 7, 437-443 (2005).

36. S. Remington, G. Wiegand, R. Huber, Crystallographic refinement and atomic

37. H. Lauble, M. C. Kennedy, H. Beinert, C. D. Stout, Crystal structures of aconitase with isocitrate and nitroisocitrate bound. Biochemistry 31, 27352748 (1992).

38. A. D. . Chapman, A. Cortés, T. . Dafforn, A. . Clarke, R. . Brady, Structural basis of substrate specificity in malate dehydrogenases: crystal structure of a ternary complex of porcine cytoplasmic malate dehydrogenase, $\alpha$ Ketomalonate and TetrahydoNAD 1 1Edited by R. Huber. J. Mol. Biol. 285, 703-712 (1999).

39. V. Yankovskaya, et al., Architecture of Succinate Dehydrogenase and Reactive Oxygen Species Generation. Science (80-. ). 299, 700-704 (2003). 
620 Theory and Experiments. Bioinformatics 27, 1575-1577 (2011).

621 41. M. J. Abraham, et al., GROMACS: High performance molecular simulations

622 through multi-level parallelism from laptops to supercomputers. SoftwareX 1-

$623 \quad 2,19-25(2015)$.

624 42. N. Schmid, et al., Definition and testing of the GROMOS force-field versions 54A7 and 54B7. Eur. Biophys. J. 40, 843-856 (2011).

626 43. N. Michaud-Agrawal, E. J. Denning, T. B. Woolf, O. Beckstein, MDAnalysis: A toolkit for the analysis of molecular dynamics simulations. J. Comput. Chem. 32, 2319-2327 (2011).

44. P. J. A. Cock, et al., Biopython: freely available Python tools for computational molecular biology and bioinformatics. Bioinformatics 25, 1422-1423 (2009). 